\title{
Effect of Educational Training Program on Research Activities among First Grade Medical Students at Zagazig University
}

\author{
Abd El-Lateef S. Ali, Sohair A. Hagag, Alaa S. Nouh*, Hanaa A. Nofal \\ Department of Public Health and community medicine, Faculty of Medicine, Zagazig University,Egypt \\ *Corresponding author: Alaa Nouh, Mobil: (+20)01097748986, Email: nouh.alaa@yahoo.com
}

\begin{abstract}
Background: One of the most important measures of scientific progress in any country in the present days is the research situation. Medical students should be equipped with ample skills and knowledge about conducting research to become an efficient researcher. Objective: This study aimed to upgrade scientific research among medical students of Faculty of Medicine, Zagazig University to assess the effect of educational training program on knowledge, attitude and skills of research. Patients and Methods: A randomized-controlled trial (an interventional study) was conducted among 110 first grade medical students. The sample was divided randomly into 2 groups: Intervention group included 55 students received the educational training program and control group that included 55 students. This study done on 3 phases: In the pre-intervention phase: knowledge, attitude and skills of research were assessed among all studied sample. Then the intervention group underwent the educational training program. Lastly in the post-intervention phase; knowledge, attitude and skills of research were assessed among both groups. Results: There was statistically significant difference of knowledge, attitude and skills of research between intervention group and control group at post-intervention phase $(\mathrm{P}<0.05)$. Also, there was statistically significant difference of knowledge, attitude and skills in intervention group preand post-intervention $(\mathrm{P}<0.05)$. Conclusion: The educational training program was effective in improving levels of knowledge, attitude and skills of research among the first grade medical students.
\end{abstract}

Keywords: Research skills, Training program, Medical students.

\section{INTRODUCTION}

Research is a systematic process to achieve new knowledge, science or invention by using of standard methods ${ }^{(1)}$. One of the most important measures of scientific progress in any country in the present days is the research situation. Therefore, the importance of conducting scientific and accurate research has increased in most countries, both developed and developing. This trend may be due to the desire to resolve the health care problems in their communities, to establish independence from other countries or to compete with them ${ }^{(2)}$.

Research training is a vital constituent of medical education, so becoming a consumer of research should be a goal for all medical students and graduates (3). The level of knowledge has also been found to be poor among these students. However, after receiving training on research a significant improvement in knowledge and a desirable change in attitude has been observed $^{(4)}$. To become an efficient researcher, one has to be equipped with ample skills and knowledge about the research methodology ${ }^{(5)}$. This study aimed to upgrade scientific research among medical students of Faculty of Medicine, Zagazig University to assess the effect of educational training program on knowledge, attitude and skills of research.

\section{PATIENTS AND METHODS}

Randomized-controlled trial ( an interventional study) was conducted at Faculty of Medicine, Zagazig University from April 2019 till October 2020 among first grade medical students, Faculty of Medicine Zagazig University. The sample was divided into 2 groups: Intervention group (55 students) received the educational training program and control group (55 students). Assuming that knowledge score in intervention group is $70 \pm 22$ and in control group $60 \pm$ 12 with confidence level $95 \%$ and power of test $80.0 \%$ . The sample size was calculated to be 110 students by using Epi program.

The students included in the study were selected by simple random sample. Then, they were classified randomly into intervention group and control group.

1st phase: knowledge, attitude and skills of research were assessed in both groups.002

2nd phase (Education session phase): Education procedures were implemented to establish objectives of the study It is done by :

- A brochure that was distributed as handout to students of intervention group.

- Lectures (power point presentation): contain research definition \& process, study designs and research methodology and guidelines for protocol and papers writing.

- Training on searching skills: Students were trained online on how to find medical websites, how to write the query and number of key words, specification and narrowing of research topics.

3rd phase (post-test phase): Reassessment of Knowledge and attitude after one month from giving the educational message. Reassessment of skills by check list for the online searching skills and using web for finding information.

Tools of study: Data of the study were collected through out the following tools:

Tool I: Structured questionnaire used to collect personal data such as sex, type of school, educational level and work of parents 
Tool II: Structured questionnaire included the following parts that were used to assess knowledge through true and false questions: components of a research, hypothesis, objectives, sample technique, pilot, methodology, the results section and references.

Scoring: In true and false questions of knowledge a correct answer was given score (1) and wrong answer was given score (0). Knowledge questions were 43 so minimum score was (0) and maximum score was (43). Knowledge of scientific research was considerd satisfactory $\geq 70 \%$, un satisfactory $<70 \%$.

Tool III: Structured questionnaire that was used to assess attitude about research. Questions were 16, each question 3 points likert scale (totally agree, no comment and disagree) ${ }^{(2)}$. Scoring: Totally agree will be given score (3), No comment will be given score (2), disagree will be given score (1). So total score was 16 x $3=48$. Attitude for scientific research was considerd positive if $\geq 70 \%$ and negative if $<70 \%$.

Tool IV : Observetional online check list to assess students skills during research as: entery on medical websites, how they write the query and number of key words, specification, narrowing of research topics and using Boolean operators.

Scoring: Skills questions were 6. So, total score was 6 . Minimum score was (0) and maximum score was (6). Skills of research was considerd competent if $\geq 70 \%$ and uncompetent if $<70 \%$.

\section{Ethical consent:}

An approval of the study was obtained from Zagazig University Academic and Ethical
Committee. Every patient signed an informed written consent for acceptance of the study. This work has been carried out in accordance with the Code of Ethics of the World Medical Association (Declaration of Helsinki) for studies involving humans.

\section{Statistical Analysis}

All data were collected, tabulated and statistically analyzed using SPSS 20.0 for windows (SPSS Inc., Chicago, IL, USA). Quantitative data were expressed as the mean \pm SD \& range, and qualitative data were expressed as percentage. $t$ test was used to compare between two groups of normally distributed variables. Mann Whitney U test was used to compare between two groups of non-normally distributed variables. F-test (ANOVA) was used to compare between more than two groups of normally distributed variables, and least significant difference was used to detect significance between groups. While, Kruskall Wallis test was used to compare between more than two groups of non-normally distributed variables. Paired $t$ test was used to compare between two dependent variables of normally distributed variables. Wilcoxon Signed Ranks Test was used to compare between two dependent variables of non-normally distributed variables. Percent of categorical variables were compared using Chi-square test or Fisher Exact test when appropriate. McNemar was used in qualitative paired data. $\mathrm{P}$ value $\leq 0.05$ was considered significant.

\section{RESULTS}

Table (1): Sociodemographic characteristics of the studied groups (each group $n=55$ )

\begin{tabular}{|c|c|c|c|c|c|c|}
\hline \multirow{2}{*}{ Variables } & \multicolumn{2}{|c|}{ Intervention group (55) } & \multicolumn{2}{|c|}{ Control group (55) } & \multirow{2}{*}{$\begin{array}{l}\chi^{2} \\
\text { test }\end{array}$} & \multirow{2}{*}{$P$ value } \\
\hline & n. & $\%$ & n. & $\%$ & & \\
\hline $\begin{array}{l}\text { Sex } \\
\text { Male } \\
\text { Female } \\
\end{array}$ & $\begin{array}{l}27 \\
28 \\
\end{array}$ & $\begin{array}{l}49.1 \\
50.9 \\
\end{array}$ & $\begin{array}{l}29 \\
26 \\
\end{array}$ & $\begin{array}{l}52.7 \\
47.3 \\
\end{array}$ & 0.14 & 0.7 \\
\hline $\begin{array}{l}\text { School } \\
\text { Governmental } \\
\text { Private }\end{array}$ & $\begin{array}{l}45 \\
10\end{array}$ & $\begin{array}{l}81.8 \\
18.2\end{array}$ & $\begin{array}{c}46 \\
9\end{array}$ & $\begin{array}{l}83.6 \\
16.4\end{array}$ & 0.064 & 0.8 \\
\hline $\begin{array}{l}\text { Education of father: } \\
\text { Secondary school } \\
\text { University } \\
\text { Postgraduate }\end{array}$ & $\begin{array}{l}18 \\
19 \\
18\end{array}$ & $\begin{array}{l}32.7 \\
34.6 \\
32.7\end{array}$ & $\begin{array}{l}19 \\
18 \\
18\end{array}$ & $\begin{array}{l}34.6 \\
32.7 \\
32.7\end{array}$ & 0.054 & 0.9 \\
\hline $\begin{array}{l}\text { Education of mother: } \\
\text { Secondary school } \\
\text { University } \\
\text { Postgraduate }\end{array}$ & $\begin{array}{l}21 \\
17 \\
17\end{array}$ & $\begin{array}{l}38.2 \\
30.9 \\
30.9 \\
\end{array}$ & $\begin{array}{l}16 \\
21 \\
18\end{array}$ & $\begin{array}{l}29.1 \\
38.2 \\
32.7 \\
\end{array}$ & 1.13 & 0.57 \\
\hline $\begin{array}{l}\text { Work of father: } \\
\text { private } \\
\text { handworker } \\
\text { professional } \\
\end{array}$ & $\begin{array}{l}18 \\
19 \\
18\end{array}$ & $\begin{array}{l}32.7 \\
34.6 \\
32.7\end{array}$ & $\begin{array}{l}19 \\
18 \\
18\end{array}$ & $\begin{array}{l}34.6 \\
32.7 \\
32.7\end{array}$ & 0.05 & 0.9 \\
\hline $\begin{array}{l}\text { Work of mother: } \\
\text { Not working } \\
\text { Working }\end{array}$ & $\begin{array}{l}16 \\
39 \\
\end{array}$ & $\begin{array}{l}29.1 \\
70.9 \\
\end{array}$ & $\begin{array}{l}19 \\
36 \\
\end{array}$ & $\begin{array}{l}34.5 \\
65.5 \\
\end{array}$ & 0.37 & 0.53 \\
\hline
\end{tabular}

$* \chi 2=$ chi square test. $(\mathrm{P} \geq 0.05=$ insignificant $)$ 
Table (1) showed that there was statistically non-significant difference between both groups regarding their sociodemographic characteristics $(\mathrm{P} \geq 0.05)$.

Table (2): Comparison between pre- and post-intervention program as regards research knowledge level among the intervention group $(\mathrm{n}=55)$

\begin{tabular}{|c|c|c|c|c|c|}
\hline \multirow{3}{*}{ Variable } & \multicolumn{4}{|c|}{ Knowledge level } & \multirow{3}{*}{ P-value } \\
\hline & \multicolumn{2}{|c|}{ Pre } & \multicolumn{2}{|c|}{ Post } & \\
\hline & $\mathbf{N}$ & $\%$ & $\mathbf{N}$ & $\%$ & \\
\hline \multirow{2}{*}{$\begin{array}{l}\text { Components of research protocol: } \\
\text { Good } \\
\text { Poor } \\
\text { Mean } \pm \text { SD } \\
\end{array}$} & $\begin{array}{c}7 \\
48\end{array}$ & $\begin{array}{l}(12.7) \\
(87.3)\end{array}$ & $\begin{array}{l}36 \\
19\end{array}$ & $\begin{array}{l}(65.5) \\
(34.5)\end{array}$ & \multirow[t]{2}{*}{$<0.001$} \\
\hline & \multicolumn{2}{|c|}{$1.9 \pm 1.2$} & \multicolumn{2}{|c|}{$3.7 \pm 1.2$} & \\
\hline \multirow{2}{*}{$\begin{array}{l}\text { Contents of abstract: } \\
\text { Good } \\
\text { Poor } \\
\text { Mean } \pm \text { SD } \\
\end{array}$} & $\begin{array}{c}6 \\
49 \\
\end{array}$ & $\begin{array}{l}(10.9) \\
(89.1)\end{array}$ & $\begin{array}{l}40 \\
15 \\
\end{array}$ & $\begin{array}{l}(72.7) \\
(27.3)\end{array}$ & \multirow[t]{2}{*}{$<0.001$} \\
\hline & \multicolumn{2}{|c|}{$1.6 \pm 1.3$} & \multicolumn{2}{|c|}{$4 \pm 1.1$} & \\
\hline \multirow{2}{*}{$\begin{array}{l}\text { Aim of the study: } \\
\text { Good } \\
\text { Poor } \\
\text { Mean } \pm \text { SD }\end{array}$} & $\begin{array}{c}3 \\
52\end{array}$ & $\begin{array}{c}(5.5) \\
(94.5)\end{array}$ & $\begin{array}{l}30 \\
25\end{array}$ & $\begin{array}{l}(54.5) \\
(45.5)\end{array}$ & \multirow[t]{2}{*}{$<0.001$} \\
\hline & \multicolumn{2}{|c|}{$0.7 \pm 0.8$} & \multicolumn{2}{|c|}{$2.2 \pm 0.8$} & \\
\hline \multirow{2}{*}{$\begin{array}{l}\text { Objective of research should be: } \\
\text { Good } \\
\text { Poor } \\
\text { Mean } \pm \text { SD }\end{array}$} & $\begin{array}{c}3 \\
52\end{array}$ & $\begin{array}{c}(5.5 \%) \\
(94.5 \%)\end{array}$ & $\begin{array}{c}47 \\
8\end{array}$ & $\begin{array}{l}(85.5) \\
(14.5)\end{array}$ & \multirow[t]{2}{*}{$<0.001$} \\
\hline & \multicolumn{2}{|c|}{$1.3 \pm 1.2$} & \multicolumn{2}{|c|}{$4.3 \pm 1.2$} & \\
\hline \multirow{2}{*}{$\begin{array}{l}\text { Probability Sample include: } \\
\text { Good } \\
\text { Poor } \\
\text { Mean } \pm \text { SD }\end{array}$} & $\begin{array}{c}1 \\
54 \\
\end{array}$ & $\begin{array}{c}(1.8) \\
(98.2) \\
\end{array}$ & $\begin{array}{l}42 \\
13 \\
\end{array}$ & $\begin{array}{l}(76.4) \\
(23.6) \\
\end{array}$ & \multirow[t]{2}{*}{$<0.001$} \\
\hline & \multicolumn{2}{|c|}{$1.2 \pm 1.1$} & \multicolumn{2}{|c|}{$3.9 \pm 1.3$} & \\
\hline \multirow{2}{*}{$\begin{array}{l}\text { Uses of a pilot study: } \\
\text { Good } \\
\text { Poor } \\
\text { Mean } \pm \text { SD } \\
\end{array}$} & $\begin{array}{l}6 \\
49 \\
\end{array}$ & $\begin{array}{l}(10.9) \\
(89.1)\end{array}$ & $\begin{array}{l}31 \\
24 \\
\end{array}$ & $\begin{array}{l}(56.4) \\
(43.6)\end{array}$ & \multirow[t]{2}{*}{$<0.001$} \\
\hline & \multicolumn{2}{|c|}{$0.63 \pm 0.67$} & \multicolumn{2}{|c|}{$1.5 \pm 0.6$} & \\
\hline \multirow{2}{*}{$\begin{array}{l}\text { Definition of research hypothesis: } \\
\text { Good } \\
\text { Poor } \\
\text { Mean } \pm \text { SD }\end{array}$} & $\begin{array}{c}2 \\
53\end{array}$ & $\begin{array}{c}(3.6) \\
(96.4)\end{array}$ & $\begin{array}{l}35 \\
20\end{array}$ & $\begin{array}{l}(63.6) \\
(36.4)\end{array}$ & \multirow[t]{2}{*}{$<0.001$} \\
\hline & \multicolumn{2}{|c|}{$1 \pm 1.1$} & \multicolumn{2}{|c|}{$2.9 \pm 1$} & \\
\hline \multirow{2}{*}{$\begin{array}{l}\text { result of a research contain } \\
\text { Good } \\
\text { Poor } \\
\text { Mean } \pm \text { SD } \\
\end{array}$} & $\begin{array}{c}4 \\
51 \\
\end{array}$ & $\begin{array}{c}(7.3) \\
(92.7) \\
\end{array}$ & $\begin{array}{l}30 \\
25 \\
\end{array}$ & $\begin{array}{c}(54.5) \\
45.5) \\
\end{array}$ & $<0.001$ \\
\hline & & & & & \\
\hline $\begin{array}{l}\text { Programs used for writing reference are } \\
\text { Good } \\
\text { Poor }\end{array}$ & $\begin{array}{c}4 \\
51 \\
\end{array}$ & $\begin{array}{c}(7.3) \\
(92.7)\end{array}$ & $\begin{array}{l}32 \\
23 \\
\end{array}$ & $\begin{array}{l}(58.2) \\
(41.8)\end{array}$ & $<0.001$ \\
\hline Mean \pm SD & & & & & \\
\hline $\begin{array}{l}\text { What is the types of epidemiological studies? } \\
\text { Good } \\
\text { Poor }\end{array}$ & $\begin{array}{c}5 \\
50\end{array}$ & $\begin{array}{c}(9.1) \\
(90.9)\end{array}$ & $\begin{array}{l}32 \\
23\end{array}$ & $\begin{array}{l}(58.2) \\
(41.8)\end{array}$ & $<0.001$ \\
\hline Mean \pm SD & & & & & \\
\hline $\begin{array}{l}\text { What is study design suitable for the } \\
\text { following? } \\
\text { Good }\end{array}$ & $\begin{array}{c}4 \\
51\end{array}$ & $\begin{array}{c}(7.3) \\
(92.7)\end{array}$ & $\begin{array}{l}31 \\
24\end{array}$ & $\begin{array}{l}(56.4) \\
(43.6)\end{array}$ & $<0.001$ \\
\hline $\begin{array}{l}\text { Poor } \\
\text { Mean } \pm \text { SD } \\
\end{array}$ & & & & & \\
\hline
\end{tabular}

*test of significance $=$ Mc Nemar $(\mathrm{P}<0.05=$ significant $)$

Table (2) showed statistically significant difference between pre- and post-intervention regarding all parameters of knowledge about research among first grade medical students $(\mathrm{P}<0.001)$. 
Table (3): Comparison between intervention group and control group regarding their total knowledge about research pre- and post-intervention

\begin{tabular}{|c|c|c|c|c|c|c|c|c|}
\hline \multirow{3}{*}{ Knowledge } & \multicolumn{4}{|c|}{ Studied groups } & \multirow{2}{*}{\multicolumn{2}{|c|}{ Pre* }} & \multirow{2}{*}{\multicolumn{2}{|c|}{ Post* }} \\
\hline & \multicolumn{2}{|c|}{$\begin{array}{l}\text { Intervention group } \\
(\mathbf{n} .=55)\end{array}$} & \multicolumn{2}{|c|}{$\begin{array}{c}\text { Control group } \\
(\mathbf{n} .=55)\end{array}$} & & & & \\
\hline & Pre & Post & Pre & post & $\mathbf{U}$ & $\mathbf{P}$ & $\mathbf{T}$ & $\mathbf{P}$ \\
\hline Mean \pm SD & $11 \pm 6.8$ & $32 \pm 6.6$ & $9 \pm 3.6$ & $16 \pm 4.6$ & \multirow{3}{*}{1.8} & \multirow{3}{*}{0.064} & \multirow{3}{*}{14.3} & \multirow{3}{*}{$<0.001$} \\
\hline $\mathbf{W}$ & \multicolumn{2}{|c|}{6.37} & \multicolumn{2}{|c|}{5.50} & & & & \\
\hline P-value & \multicolumn{2}{|c|}{$<0.001$} & \multicolumn{2}{|c|}{$<0.001$} & & & & \\
\hline$\%$ of change & \multicolumn{2}{|c|}{$180 \%$} & \multicolumn{2}{|c|}{$75.88 \%$} & \multicolumn{2}{|c|}{$19 \%$} & \multicolumn{2}{|c|}{$65 \%$} \\
\hline
\end{tabular}

U= Mann-whitney. W=Wilcoxon ranked sig test. $\quad(\mathrm{P} \geq 0.05=$ insignificant $) \quad(\mathrm{P}<0.05=$ significant $)$

(pre*=Intervention and control groups pre intervention phase) (post*= Intervention and control groups post intervention training sessions)

There was statistically significant difference of knowledge in intervention group pre- and post- intervention and also in control group pre- and post-intervention. Additionally the percent of improvement of knowledge score in the intervention group was $180 \%$ versus $75.8 \%$ in the control group.

Table (4): Comparison between pre- and post-intervention program regarding attitude among the intervention group (n $=55$ )

\begin{tabular}{|c|c|c|c|c|c|}
\hline \multirow{3}{*}{ Variable } & \multicolumn{4}{|c|}{ Attitude } & \multirow{3}{*}{ P-value } \\
\hline & \multicolumn{2}{|c|}{ Pre } & \multicolumn{2}{|c|}{ Post } & \\
\hline & n. & $\%$ & n. & $\%$ & \\
\hline $\begin{array}{l}\text { Each student should participate in research : } \\
\text { Totally agree } \\
\text { No comment } \\
\text { Disagree }\end{array}$ & $\begin{array}{c}16 \\
7 \\
32\end{array}$ & $\begin{array}{l}29.1 \\
12.7 \\
58.2\end{array}$ & $\begin{array}{c}42 \\
9 \\
4\end{array}$ & $\begin{array}{c}76.4 \\
16.3 \\
7.3\end{array}$ & $<0.001$ \\
\hline $\begin{array}{l}\text { Conducting a research is easy : } \\
\text { Totally agree } \\
\text { No comment } \\
\text { Disagree }\end{array}$ & $\begin{array}{c}14 \\
5 \\
36\end{array}$ & $\begin{array}{c}25.5 \\
9.1 \\
65.4\end{array}$ & $\begin{array}{c}38 \\
6 \\
11\end{array}$ & $\begin{array}{l}69.1 \\
10.9 \\
20.0\end{array}$ & $<0.001$ \\
\hline $\begin{array}{l}\text { I like to participate in research : } \\
\text { Totally agree } \\
\text { No comment } \\
\text { Disagree }\end{array}$ & $\begin{array}{c}15 \\
6 \\
34\end{array}$ & $\begin{array}{l}27.3 \\
10.9 \\
61.8\end{array}$ & $\begin{array}{c}44 \\
1 \\
10\end{array}$ & $\begin{array}{c}80.0 \\
1.8 \\
18.2\end{array}$ & $<0.001$ \\
\hline $\begin{array}{l}\text { I tend to perform research within the community : } \\
\text { Totally agree } \\
\text { No comment } \\
\text { Disagree }\end{array}$ & $\begin{array}{c}14 \\
3 \\
38\end{array}$ & $\begin{array}{c}25.5 \\
5.4 \\
69.1\end{array}$ & $\begin{array}{c}39 \\
6 \\
10\end{array}$ & $\begin{array}{l}70.9 \\
10.9 \\
18.2\end{array}$ & $<0.001$ \\
\hline $\begin{array}{l}\text { Performing research is important for me to become a } \\
\text { specialist: } \\
\text { Totally agree } \\
\text { No comment } \\
\text { Disagree }\end{array}$ & $\begin{array}{c}17 \\
6 \\
32\end{array}$ & $\begin{array}{l}30.9 \\
10.9 \\
58.2\end{array}$ & $\begin{array}{c}42 \\
8 \\
5\end{array}$ & $\begin{array}{c}76.4 \\
14.5 \\
9.1\end{array}$ & $<0.001$ \\
\hline $\begin{array}{l}\text { Skills that I gain during research are useful in my future } \\
\text { work: } \\
\text { Totally agree } \\
\text { No comment } \\
\text { Disagree }\end{array}$ & $\begin{array}{c}17 \\
5 \\
33\end{array}$ & $\begin{array}{c}30.9 \\
9.1 \\
60.0\end{array}$ & $\begin{array}{c}41 \\
4 \\
10\end{array}$ & $\begin{array}{c}74.5 \\
7.3 \\
18.2\end{array}$ & $<0.001$ \\
\hline $\begin{array}{l}\text { Taking time to research is time wasted, if it doesn't enhance } \\
\text { my future career: } \\
\text { Totally agree } \\
\text { No comment } \\
\text { Disagree }\end{array}$ & $\begin{array}{c}15 \\
8 \\
32\end{array}$ & $\begin{array}{l}27.3 \\
14.5 \\
58.2\end{array}$ & $\begin{array}{c}40 \\
5 \\
10\end{array}$ & $\begin{array}{c}72.7 \\
9.1 \\
18.2\end{array}$ & $<0.001$ \\
\hline $\begin{array}{l}\text { Research should be offered in training to all students in } \\
\text { studies classes: }\end{array}$ & 16 & 29.1 & 36 & 65.5 & $<0.001$ \\
\hline
\end{tabular}




\begin{tabular}{|c|c|c|c|c|c|}
\hline \multirow{3}{*}{ Variable } & \multicolumn{4}{|c|}{ Attitude } & \multirow{3}{*}{ P-value } \\
\hline & \multicolumn{2}{|c|}{ Pre } & \multicolumn{2}{|c|}{ Post } & \\
\hline & n. & $\%$ & n. & $\%$ & \\
\hline $\begin{array}{l}\text { Totally agree } \\
\text { No comment } \\
\text { Disagree }\end{array}$ & $\begin{array}{c}5 \\
34\end{array}$ & $\begin{array}{c}9.1 \\
61.8\end{array}$ & $\begin{array}{c}2 \\
17\end{array}$ & $\begin{array}{c}3.6 \\
30.9\end{array}$ & \\
\hline $\begin{array}{l}\text { I would like to replace another class related to my field with a } \\
\text { research class: } \\
\text { Totally agree } \\
\text { No comment } \\
\text { Disagree }\end{array}$ & $\begin{array}{c}11 \\
5 \\
39\end{array}$ & $\begin{array}{c}20.0 \\
9.1 \\
70.9\end{array}$ & $\begin{array}{c}37 \\
2 \\
16\end{array}$ & $\begin{array}{c}67.3 \\
3.6 \\
29.1\end{array}$ & $<0.001$ \\
\hline $\begin{array}{l}\text { Education on research should be compulsory in the student } \\
\text { curriculum: } \\
\text { Totally agree } \\
\text { No comment } \\
\text { Disagree }\end{array}$ & $\begin{array}{l}13 \\
11 \\
31\end{array}$ & $\begin{array}{l}23.6 \\
20.0 \\
56.4\end{array}$ & $\begin{array}{c}42 \\
13\end{array}$ & $\begin{array}{c}76.4 \\
23.6\end{array}$ & $<0.001$ \\
\hline $\begin{array}{l}\text { Research is beneficial, because it improves critical thinking: } \\
\text { Totally agree } \\
\text { No comment } \\
\text { Disagree }\end{array}$ & $\begin{array}{c}14 \\
8 \\
33\end{array}$ & $\begin{array}{l}25.5 \\
14.5 \\
60.0\end{array}$ & $\begin{array}{c}43 \\
3 \\
9\end{array}$ & $\begin{array}{c}78.1 \\
5.5 \\
16.4\end{array}$ & $<0.001$ \\
\hline $\begin{array}{l}\text { Research is useful, because it helps to change policies: } \\
\text { Totally agree } \\
\text { No comment } \\
\text { Disagree }\end{array}$ & $\begin{array}{l}12 \\
11 \\
32 \\
\end{array}$ & $\begin{array}{l}21.8 \\
20.0 \\
58.2\end{array}$ & $\begin{array}{c}41 \\
5 \\
9 \\
\end{array}$ & $\begin{array}{c}74.5 \\
9.1 \\
16.4\end{array}$ & $<0.001$ \\
\hline $\begin{array}{l}\text { I wish to publish the results of some research: } \\
\text { Totally agree } \\
\text { No comment } \\
\text { Disagree }\end{array}$ & $\begin{array}{c}15 \\
7 \\
33\end{array}$ & $\begin{array}{c}27.3 \\
12.7 \\
60.0\end{array}$ & $\begin{array}{c}41 \\
2 \\
12\end{array}$ & $\begin{array}{r}74.5 \\
3.7 \\
21.8\end{array}$ & $<0.001$ \\
\hline $\begin{array}{l}\text { Research methodology workshops at the university will be } \\
\text { very effective for me: } \\
\text { Totally agree } \\
\text { No comment } \\
\text { Disagree }\end{array}$ & $\begin{array}{c}12 \\
8 \\
35\end{array}$ & $\begin{array}{r}21.8 \\
14.5 \\
63.7\end{array}$ & $\begin{array}{r}37 \\
4 \\
14\end{array}$ & $\begin{array}{r}67.3 \\
7.2 \\
25.5 \\
\end{array}$ & $<0.001$ \\
\hline $\begin{array}{l}\text { Research is essential for improving health care of patients: } \\
\text { Totally agree } \\
\text { No comment } \\
\text { Disagree }\end{array}$ & $\begin{array}{c}20 \\
6 \\
29\end{array}$ & $\begin{array}{c}36.4 \\
10.9 \\
52.7\end{array}$ & $\begin{array}{c}42 \\
1 \\
12\end{array}$ & $\begin{array}{c}76.4 \\
1.8 \\
21.8\end{array}$ & $<0.001$ \\
\hline $\begin{array}{l}\text { Research improve communication skills: } \\
\text { Totally agree } \\
\text { No comment } \\
\text { Disagree }\end{array}$ & $\begin{array}{r}12 \\
6 \\
37 \\
\end{array}$ & $\begin{array}{l}21.8 \\
10.9 \\
67.3 \\
\end{array}$ & $\begin{array}{r}37 \\
8 \\
10 \\
\end{array}$ & $\begin{array}{r}67.3 \\
14.5 \\
18.2 \\
\end{array}$ & $<0.001$ \\
\hline
\end{tabular}

*Test: Chi square test

Table (4) showed statistically significant difference between pre- and post-intervention regarding all parameters of attitude about research among first grade medical students $(\mathrm{P}<0.001)$. 
Table (5): Comparison between intervention group and control group regarding their total attitude about research preand post-intervention

\begin{tabular}{|c|c|c|c|c|c|c|c|c|}
\hline \multirow{3}{*}{ Attitude } & \multicolumn{4}{|c|}{ Studied groups } & \multirow{2}{*}{\multicolumn{2}{|c|}{ Pre* }} & \multirow{2}{*}{\multicolumn{2}{|c|}{ Post* }} \\
\hline & \multicolumn{2}{|c|}{$\begin{array}{l}\text { Intervention } \\
\text { group }(\mathbf{n} .=55)\end{array}$} & \multicolumn{2}{|c|}{$\begin{array}{c}\text { Control group } \\
(\mathbf{n} .=55)\end{array}$} & & & & \\
\hline & Pre & Post & Pre & post & $\mathbf{t}$ & $\mathbf{P}$ & $\mathbf{T}$ & $\mathbf{P}$ \\
\hline Mean \pm SD & $26.4 \pm 3.9$ & $40.5 \pm 6.6$ & $26.3 \pm 3.9$ & $26.9 \pm 8.3$ & & & & \\
\hline Paired t-test & \multicolumn{2}{|c|}{13.8} & \multicolumn{2}{|c|}{0.44} & 0.09 & 0.9 & 9.5 & $<0.001$ \\
\hline P-value & \multicolumn{2}{|c|}{$<0.001$} & \multicolumn{2}{|c|}{0.65} & & & & \\
\hline$\%$ of Change & \multicolumn{2}{|c|}{$53 \%$} & \multicolumn{2}{|c|}{$2 \%$} & \multicolumn{2}{|c|}{$0.2 \%$} & \multicolumn{2}{|c|}{$40 \%$} \\
\hline
\end{tabular}

$(\mathrm{P} \geq 0.05=$ insignificant $) \quad(\mathrm{P}<0.05=$ significant $) \quad$ (pre*=Intervention and control groups pre intervention phase $)$ (post* $=$ Intervention and control groups post intervention training sessions)

Table (5) showed statistically significant difference of attitude in intervention group pre- and post-intervention but there was statistically non-significant difference in control group pre- and post- intervention. Additionally the percent of change of attitude in the intervention group was $53 \%$ versus $2 \%$ in the control group.

Table (6): Comparison between pre- and post-intervention program regarding skills among the intervention group $(\mathrm{n}=55)$

\begin{tabular}{|c|c|c|c|c|c|}
\hline \multirow{3}{*}{ Variable } & \multicolumn{4}{|c|}{ Skills } & \multirow{3}{*}{ P-value } \\
\hline & \multicolumn{2}{|c|}{ Pre } & \multicolumn{2}{|c|}{ Post } & \\
\hline & n. & $\%$ & n. & $\%$ & \\
\hline $\begin{array}{l}\text { Step 1: Find search engine or medical website } \\
\text { Done } \\
\text { Not Done }\end{array}$ & $\begin{array}{l}17 \\
38 \\
\end{array}$ & $\begin{array}{l}30.9 \\
69.1 \\
\end{array}$ & $\begin{array}{c}51 \\
4 \\
\end{array}$ & $\begin{array}{c}92.7 \\
7.3 \\
\end{array}$ & $<0.001$ \\
\hline $\begin{array}{l}\text { Step 2: Write keywords or query } \\
\text { Done } \\
\text { Not done }\end{array}$ & $\begin{array}{l}11 \\
44 \\
\end{array}$ & $\begin{array}{l}20.0 \\
80.0\end{array}$ & $\begin{array}{l}43 \\
12 \\
\end{array}$ & $\begin{array}{l}78.2 \\
21.8 \\
\end{array}$ & $<0.001$ \\
\hline $\begin{array}{l}\text { Step 3: use more than } 3 \text { key words : } \\
\text { Done } \\
\text { Not done }\end{array}$ & $\begin{array}{c}7 \\
48 \\
\end{array}$ & $\begin{array}{l}12.7 \\
87.3\end{array}$ & $\begin{array}{c}46 \\
9 \\
\end{array}$ & $\begin{array}{l}83.6 \\
16.4\end{array}$ & $<0.001$ \\
\hline $\begin{array}{l}\text { Step 4: use phrases enclosed by quotation marks: } \\
\text { Done } \\
\text { Not done }\end{array}$ & $\begin{array}{c}1 \\
54\end{array}$ & $\begin{array}{c}1.8 \\
98.2\end{array}$ & $\begin{array}{l}43 \\
12\end{array}$ & $\begin{array}{l}78.2 \\
21.8\end{array}$ & $<0.001$ \\
\hline $\begin{array}{l}\text { Step 5: use And/plus between keywords: } \\
\text { Done } \\
\text { Not done }\end{array}$ & $\begin{array}{l}10 \\
45\end{array}$ & $\begin{array}{l}18.2 \\
81.8\end{array}$ & $\begin{array}{c}47 \\
8 \\
\end{array}$ & $\begin{array}{l}85.5 \\
14.5\end{array}$ & $<0.001$ \\
\hline $\begin{array}{l}\text { Step 6: se specific term } \\
\text { Done } \\
\text { Not done }\end{array}$ & $\begin{array}{c}3 \\
52 \\
\end{array}$ & $\begin{array}{c}5.5 \\
94.5\end{array}$ & $\begin{array}{c}46 \\
9\end{array}$ & $\begin{array}{l}83.6 \\
16.4\end{array}$ & $<0.001$ \\
\hline
\end{tabular}

$(\mathrm{P}<0.05=$ significant $)$

Test of significant $=\mathrm{MC}$ Nemar test .

Table (6) showed statistically significant difference between pre- and post-intervention as regards all parameters of skills of research among first grade medical students $(\mathrm{p}<0.001)$. 
Table (7): Comparison between intervention group and control group regarding their skills of research pre- and postintervention

\begin{tabular}{|c|c|c|c|c|c|c|c|c|}
\hline & \multicolumn{4}{|c|}{ Total skills } & \multirow{2}{*}{\multicolumn{2}{|c|}{ Pre* }} & \multirow{2}{*}{\multicolumn{2}{|c|}{ Post* }} \\
\hline & \multicolumn{2}{|c|}{$\begin{array}{c}\text { Intervention } \\
\text { group }(\mathbf{n} .=55)\end{array}$} & \multicolumn{2}{|c|}{$\begin{array}{c}\text { Control group } \\
(\mathbf{n} .=55)\end{array}$} & & & & \\
\hline & Pre & Post & Pre & Post & $\mathbf{U}$ & $\mathbf{P}$ & $\mathbf{U}$ & $\mathbf{P}$ \\
\hline $\begin{array}{l}\text { Mean } \\
\text { Median (range) }\end{array}$ & $\begin{array}{c}0.89 \pm 0.7 \\
1(0-2)\end{array}$ & $\begin{array}{l}5 \pm 1.1 \\
5(1-6)\end{array}$ & $\begin{array}{c}0.76 \pm 0.88 \\
1(0-4)\end{array}$ & $\begin{array}{c}0.49 \pm 0.71 \\
0(0-2)\end{array}$ & & & & \\
\hline W & \multicolumn{2}{|c|}{6.48} & \multicolumn{2}{|c|}{1.46} & 1.3 & 0.19 & 9.2 & $<0.001$ \\
\hline P-value & \multicolumn{2}{|c|}{$<0.001$} & \multicolumn{2}{|c|}{0.14} & & & & \\
\hline$\%$ of Change & \multicolumn{2}{|c|}{$460 \%$} & \multicolumn{2}{|c|}{$35 \%$} & \multicolumn{2}{|c|}{$18 \%$} & \multicolumn{2}{|c|}{$166 \%$} \\
\hline
\end{tabular}

U= Mann-Whitney. $\quad \mathrm{W}=$ Wilcoxon ranked sig test. $\quad(\mathrm{P} \geq 0.05=$ insignificant $)(\mathrm{P}<0.05=$ significant $)$

(pre*=Intervention and control groups pre intervention phase) $\quad$ (post* ${ }^{*}=$ Intervention and control groups post intervention training sessions)

Table (7) showed statistically significant difference of skills in intervention group pre- and post-intervention but there was statistically non-significant difference in control group pre- and post-intervention. Additionally the percent of change of skills in the intervention group was $460 \%$ versus $35 \%$ in the control group.

\section{DISCUSSION}

This study was performed on 110 first-year medical students divided into two groups, with 55 students in each. $51 \%$ of the interventional group was females, while $52.7 \%$ of the controls were males. The majority of students sample attended a governmental or public secondary school $(81.8 \%$ in the intervention group and $83.6 \%$ in the control group). There was no statistically significant difference between both groups regarding their sociodemographic characteristics.

The present study demonstrated lower preinterventional knowledge scores within the intervention group, compared to their consecutive scores following the implementation of the educational training program (P-value< 0.001 in all domains of knowledge assessment). This comes in concordance with Pallamparthy and Basavareddy ${ }^{(6)}$ who found that the knowledge scores among the interventional group showed significant improvement over those obtained from non-trained students. This proves the efficacy of research training courses and actively exhibits the vital role of these programs in forming an appropriate scientific background. Moreover, Al-Tannir et al. ${ }^{(7)}$ noticed that knowledge scores improved by $70 \%$ amongst undergraduate medical students who received training about research.

The current study detected significant difference between pre- and post-interventional knowledge score among the experimental group where percent of improvement was $180 \%$ (p-value < 0.001) versus $75.8 \%$ in the control group. This finding may be attributed to the fact that first grade medical students studied brief research course in their curriculum. Moreover, percent of change of knowledge scores in between the interventional and control groups in the pre-interventional phase was 19\% (p-value $=0.064)$, while in the post-interventional phase was $65 \%$ (p-value
$<0.001)$. This is in agreement with Noorelahi et al. ${ }^{(8)}$ who found that score of knowledge was higher among posttest students compared to the pretest. Also Abushouk et al. (9) found that knowledge score increased at post-intervention compared to preintervention and the difference was statistically significant. This also proves the importance of research courses and its role in upgrading research knowledge scores among medical students.

The present study demonstrated lower preinterventional attitude scores within the experimental group compared to their consecutive scores following the implementation of the educational training program (p-value< 0.001). In the current study there was a negative attitude towards research in the preinterventional set and such findings disagree with Noorelahi et al. ${ }^{(8)}$ where about $70 \%$ of the studied sample exhibited positive attitudes towards medical research without educational program. This comes in conflict also with the findings from Abushouk et al. ${ }^{(9)}$ who found that there was a positive attitude amongst non-trained Egyptian medical students at Ain Shams University. Such a discrepancy in findings can be attributed to the fact that our studied sample consisted solely of first-grade students. This could justify the poor level of positive attitudes as most students would probably have hasty views and conclusions in their first years.

The current study detected significant difference between pre- and post-interventional attitude score among the interventional group where percent of improvement was $53 \%$ (mean difference/MD $=14.1$, $\mathrm{p}$ value $<0.001)$. Meanwhile, in the control group percent of improvement in the attitude score was 2\% (Mean Diff. $=0.58$ ).

As regards the between-group analysis, no difference was observed between the pre-interventional 
scores of the two groups ( $p$-value $=0.9)$, whereas in the post-interventional phase there was statistically significant difference between experiments and controls ( $p$-value $<0.001)$. This is in conflict with Chapman et al. (10) who found that medical students were welloriented and have positive attitude about medical research from the start and there was no significant difference between intervention and control groups. These findings are thought to be resulting from increase awareness of his medical students and their career-wise agenda based on extensive competition for postgraduate positions, or due to they think that there is time attrition and consumption in clinical practice more than research activities after graduation and elevating the level of clinical or education knowledge alone may not be an adequate measure among medical students in their future careers but also research activities are important (10).

The present study demonstrated lower preinterventional skills scores within the experimental group compared to their consecutive scores following the implementation of the educational training program, (P-value $<0.001$ in all domains of skills assessment). This is in agreement with Devi et al. ${ }^{(11)}$ who found that the majority of the participating medical students $(61 \%)$ acknowledged the beneficial influence of such programs on their research skills and agreed that such programs should be mandatory in medical schools.

The current study detected significant difference between pre- and post-interventional skills score among the interventional group where percent of improvement was $460 \%$ (p-value < 0.001 ). Meanwhile, in the control group percent of improvement in the total skills score was 35\% (Mean Diff. $=0.27$ ). Moreover, the percent of change of skills scores in between the interventional and control groups in the preinterventional phase was $18 \%$ while in the postinterventional phase was $166 \%$ where a significant difference was evident ( $\mathrm{p}$-value $<0.001$ ). This is similar to Mullan et al. ${ }^{(12)}$ who reported that the medical students exhibited significantly higher skills scores after training program about research skills. In another study conducted by Black $\boldsymbol{e t}$ al. ${ }^{(13)}$, they found significant improvement in research skill scores of the postinterventional group.

\section{CONCLUSION}

The educational training program improved levels of knowledge, attitude and skills of research among the first grade medical students.

Conflict of interest: The authors declare no conflict of interest.

Funding sources: The authors have no funding to report.

\section{REFERENCES}

1. Khobragade S, Khobragade Y, Abbas L (2018): A study on knowledge, attitude and approach towards research methodology amongst Malaysian medical students. MJMS., 3 (1): 6-11.

2. Memarpour M, Fard A, Ghasemi $R$ (2015): Evaluation of attitude to, knowledge of and barriers toward research among medical science students. Asia Pacific Family Medicine, 14 (1): 1-5.

3. Ibrahim N, Fetyani D, LBashwari J (2013): Knowledge, Attitude \& Practice of medical students and interns about research with implementation of educational intervention program, King Abdulaziz University, Jeddah. Rawal Medical Journal, 38: 432439.

4. Kyaw H, than N, Lwin H et al. (2018): Knowledge, attitudes, and barriers toward research the perspectives of undergraduate medical and dental students. J Edu Health Promot., 7: 23-29.

5. Zehra N, Hassaan A, Mushtaq S (2015): Research amongst Junior and Senior Medical Students; Comparison of Knowledge, Attitude and Practice. Prof Med J., 22 (1): 121-6

6. Pallamparthy S, Basavareddy A (2019): Knowledge, attitude, practice, and barriers toward research among medical students: A cross-sectional questionnaire-based survey. Perspectives in Clinical Research, 10 (2): 73-78.

7. Al-Tannir M, Abu-Shaheen A, AlSumaih S et al. (2018): Research Knowledge and Skills Among Medical and Allied Health Students Attending a Summer Research Course: A Pretest and Posttest Analysis. Cureus, 10 (8): 3132-7.

8. Noorelahi M, Soubhanneyaz A, Kasim K (2015): Perceptions, barriers, and practices of medical research among students at Taibah College of Medicine, Madinah, Saudi Arabia. Advances in Medical Education and Practice, 6: 479-485.

9. Abushouk I, Hatata N, Omran M et al. (2016): Attitudes and Perceived Barriers among Medical Students towards Clinical Research: A Cross-Sectional Study in an Egyptian Medical School. Journal of Biomedical Education, 16: 1-7.

10. Chapman S, Glasbey J, Khatri C et al. (2015): Promoting research and audit at medical school: Evaluating the educational impact of participation in a student-led national collaborative study Career choice, professional education and development. BMC Medical Education, 15 (1): 47-53.

11. Devi V, Abraham R, Adiga A et al. (2012): Fostering research skills in undergraduate medical students through Mentored Student Projects: example from an Indian medical school. Kathmandu University Medical Journal, 31: 294-298.

12. Mullan J, Weston $K$, Rich $W$ et al. (2014): Investigating the impact of a research-based integrated curriculum on self-perceived research experiences of medical students in community placements: A pre- and post-test analysis of three student cohorts. BMC Medical Education, 14 (1): 161-166.

13. Black M, Curran M, Golshan S et al. (2013): Summer Research Training for Medical Students: Impact on Research Self-Efficacy. Clinical and Translational Science, 6 (6): 487-489. 